\title{
Reply to Reviewer Comments
}

Journal: The Journal of Physical Chemistry Letters

Manuscript ID: jz-2021-03738w

Original Submission Date: 15-Nov-2021

Title: "Exciton-Phonon Coupling and Low Energy Emission in 2D and Quasi-2D $\mathrm{BA}_{2} \mathrm{MA}_{n-1} \mathrm{~Pb}_{n} \mathrm{l}_{3 n+1}$ Thin Films with Improved Phase Purity"

Authors: Shen, Rong; Xu, Jiayi; Yao, Xiang; Guo, Zilong; Yang, Wensheng; Ma, Xiaonan

We thank two reviewers for their detailed comments that helped us improve the clarity of the presentation in the revised version of our work. We have addressed all points in detail as listed below, and we have modified the manuscript accordingly, including additional, new data, and new figures.

Reviewers' comments in this reply letter are typed in black, our response in blue, and explicit changes to the manuscript in red font. Major modifications are summarized in a List of Changes at the end of this reply letter. Furthermore, all modifications are visible in a marked-up version of the manuscript. Whenever page numbers are provided in our reply, they refer to the revised, marked-up version.

\section{Reviewer(s)' Comments to Author:}

\section{$\underline{\text { Reviewer } 1}$}

Shen et al. reported the coupling of excitons with acoustic and longitudinal phonons in LD phase pure $\mathrm{Pb}$ perovskites. The authors also presented the emissions of low-lying self-trapped excitons (STE) and bound excitons due to structural defects (DE) for the LD perovskites. The work is done systematically and the corresponding interpretation is presented logically.

We thank reviewer for the positive evaluation on our work. We've revised the manuscript comprehensively according reviewers' comments.

However, as mentioned by the authors several such works have already been reported for LD perovskites prepared from single crystals or exfoliated flakes and the results presented here are not so different from the previous works. Lack of novelty is a major drawback of this work. 
As reviewer indicated, the exciton/electron - phonon (E-P) coupling in exfoliated LD $\left.B A_{2} \mathrm{MA}_{n-1} \mathrm{~Pb}_{n}\right|_{3 n+1}$ flakes or nanocrystals have been reported previously, but there are still open questions. For instance, Tisdale et al. ( $\mathrm{J}$. Phys. Chem. Lett. 2020, 11, 8565-8572) and Li et al. (J. Phys. Chem. Lett. 2019, 10, 2546-2553) reported $n$ value dependent thermal expansion (АTE) and E-P coupling (AEP) which contribute to the temperature dependent bandgap of exfoliated LD BA$\left.2 M A_{n-1} P_{n}\right|_{3 n+1}$. However, it is puzzling that the $n$ value dependence of ATE and AEP these two works reported does not consistent with each other. Even for such fundamental issue, the debate still exists. As illustrated in Figure R1, our result provides consistent description that how layer thickness ( $n$ value) affects the E-P coupling, i.e. the bandgap (Figure R1b) and linewidth (Figure R1c) data leads to similar trend of E-P coupling.
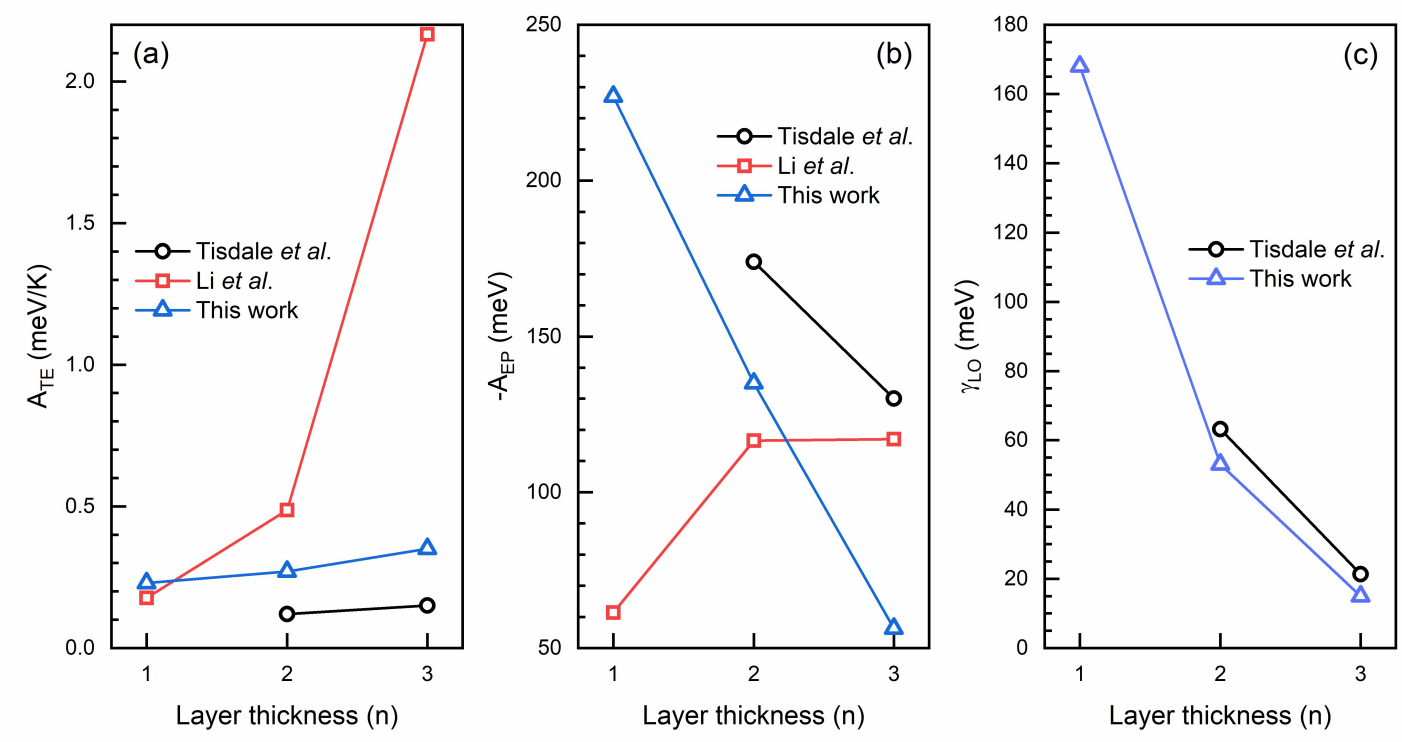

Figure R1. Comparison of estimated parameters among Tisdale et al. (JPCL 2020, 8565), Li et al. (JPCL 2019, 2546), and this work.

Moreover, the authors failed to explain clearly how they controlled the phase purity of the LD samples. I suggest the authors to consider revising their manuscript based on the following questions.

This is a critical issue regarding the sample preparation, we will discuss the corresponding details in the following part.

1) Please provide a detailed explanation of obtaining phase purity thinfilm samples. Particularly, the authors mentioned when precursors were mixed in stoichiometric ratios, they got mixed phase samples while adjusting the precursor ratios resulted in improving the phase purity. Please clearly indicate absolute ratios of precursors used for the prepared samples and also explain clearly why impure phases forms with stoichiometric proportions, whereas adjustments would lead to pure phases from a thermodynamic perspective. 
Improving phase purity of LD perovskite thin film is a complicated issue and have puzzled the community for a quite long time. In general, the solution preparation of LD perovskite thin film has inherent drawback on phase purity. During spin coating of stoichiometric precursor solution, the solvent from the liquid-air interface evaporates rapidly, which leads to unexpected high concentration of the precursor solution at the surface. Since the $n=\infty$ phase (MAPbl 3 ) has low formation energy (Nat. Commun., 2019, 10, 1008), the surface portion of films are favored to form quasi-2D phases with larger $n$ values. Meanwhile, the quasi-2D phases with lower $n$ values are correspondingly easier to form in bottom portion of films. As the result, the prepared films show broad distribution of $n$ value, i.e. poor phase purity.

Base on such consideration, we adopted two aspects of strategies to improve the phase purity of quasi-2D ( $n=2$ and 3$)$ thin films.

(i) We prepare precursor solution ( $\mathrm{MAPbl}_{3}$ and $\mathrm{BA}_{2} \mathrm{Pbl}_{4}$ ) at relatively low concentration $(0.1 \mathrm{~mol} / \mathrm{L})$. The resulted thinner films can largely eliminate the difference of phase distribution between surface and bottom part, which can be verified by room-temperature PL spectra from double sides of the quasi-2D ( $n$ $=2$ and 3 ) thin films (see Figure R2).
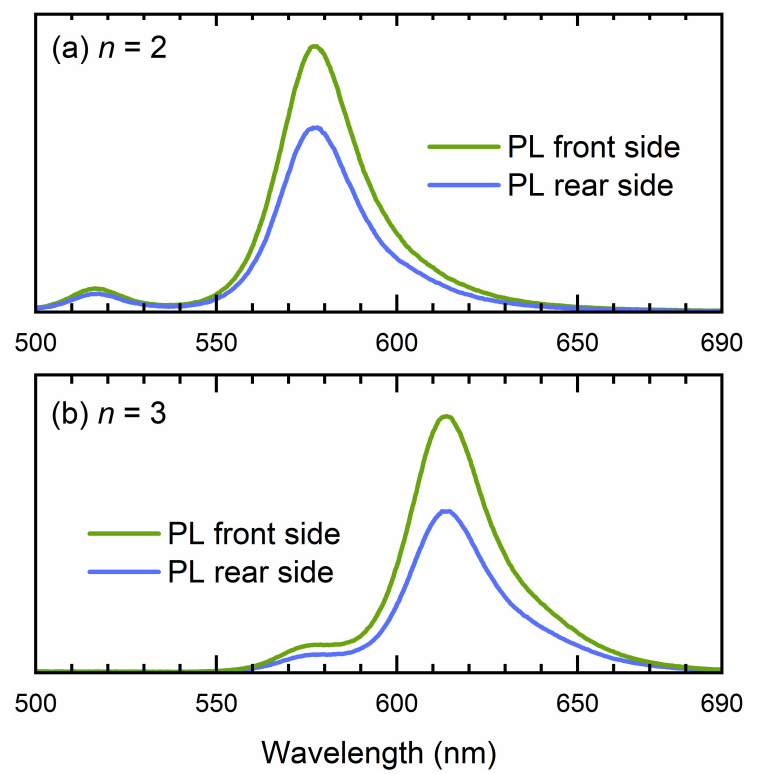

Figure R2. Room-temperature PL spectra of quasi-2D $n=2$ (a) and $n=3$ (b) films from front (green lines) and rear (blue lines) side. No phase difference can be observed between the front and rear sides of thin films, while the different PL intensity originates from the attenuation and reflection of excitation and PL on surfaces of substrates.

(ii) Furthermore, it is found that the lower MA/BA ratio than stoichiometry ( 1 for $n=2$ and 2 for $n=3$ ) can greatly suppress the formation of larger $n$ value phases during the spin coating. However, lower MA/BA ratio can also enhance 
the formation of lower $n$ value phases. We have performed systematic investigation on this issue and found that specific MA/BA ratio (0.6 for $\boldsymbol{n}=\mathbf{2}$ and $\mathbf{1 . 3}$ for $n=3$ ) can make best compromise for this dilemma and leads to improved phase purity.

However, it's worth to note that the indicated optimal MA/BA ratio (0.6 for $n=2$ and 1.3 for $n=3$ ) cannot guarantee the successful preparation of thin films with phase purity as we displayed in manuscript. In fact, under the indicated optimal MA/BA ratio, only $10-15 \%$ among the prepared parallel samples exhibit qualified phase purity (as can be seen in Figure R3). We are still working on it and trying to figure out more information for improving the manufacturing. But at this stage, we have to select thin films with qualified phase purity from parallel samples by using XRD and room-temperature PL measurements. The selected samples will be further investigated as described in the manuscript.
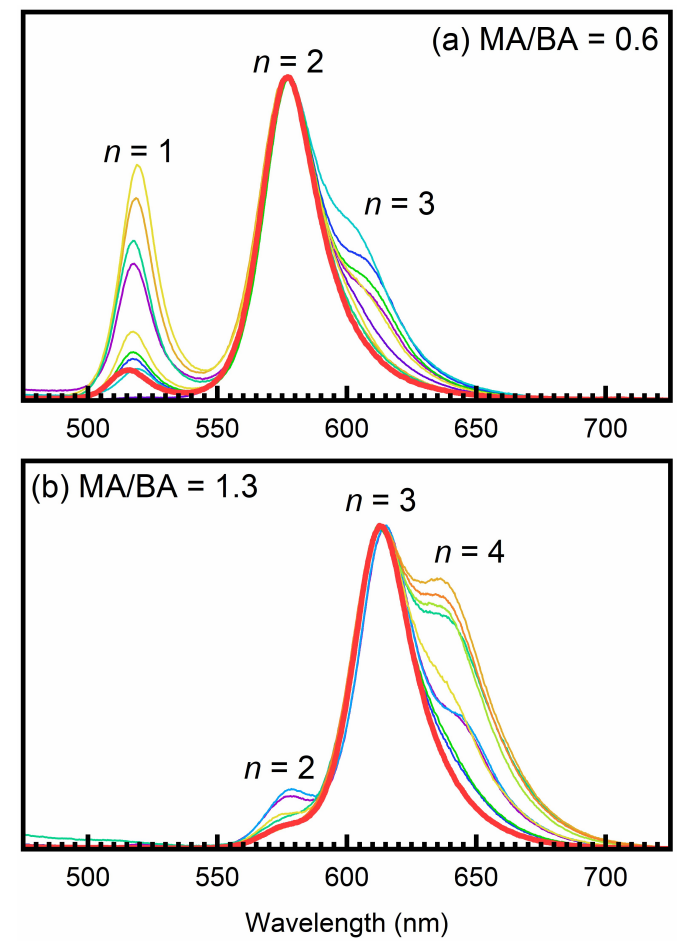

Figure R3. Room temperature PL spectra of 10 parallel prepared LD thin film samples with $M A / B A=0.6(a)$ and $1.3(b)$. The thick red lines represent PL spectra of selected samples with qualified phase purity of $n=2$ (a) and $n=3$ (b). Among all parallel samples, only $10-15 \%$ exhibits qualified phase purity in most of cases, which was selected for temperature dependent $\mathrm{PL}$ experiments as described in manuscript.

We added description on page 4 of manuscript: "The practical strategy to prepare 2D $\left(\mathrm{BA}_{2} \mathrm{Pbl}_{4}\right)$ and phase purity improved quasi-2D $\left.\mathrm{BA}_{2} \mathrm{MA}_{n-1} \mathrm{~Pb}_{n}\right|_{3 n+1}(n$ $=2$ and 3) thin films was comprehensively described in Supporting Information (Section S1). The phase purity of prepared thin films was further characterized by X-ray diffraction (XRD) and room-temperature PL spectra." 
The related details of sample preparation have been implemented in Supporting Information (Section S1) on page 3 and 4:

"During spin-coating of stoichiometric precursor solution, the solvent from the liquid-air interface evaporates rapidly, which leads to unexpected high concentration of the precursor solution at the surface. Due to the low formation energy (Nat. Commun., 2019, 10, 1008) of 3D MAPbl 3 phase $(n=\infty)$, surface portion of films are favored to form quasi-2D phases with larger $n$ values while the bottom part is dominated by phases with smaller $n$ value. As the result, the prepared films show broad distribution of $n$ value, i.e. poor phase purity."

"For improving the phase purity of prepared quasi-2D ( $n=2$ and 3 ) thin films, two aspects of strategies were adopted: 1$)$ Relatively low concentration (0.1 $\mathrm{mol} / \mathrm{L}$ ) was employed for $\mathrm{MAPbl}_{3}$ and $\mathrm{BA}_{2} \mathrm{Pbl}_{4}$ precursor solution, which largely eliminates the phase difference between surface and bottom part of thin films. 2) lower $\mathrm{MAPbl}_{3} / \mathrm{BA}_{2} \mathrm{Pbl}_{4}$ ratio than stoichiometry can greatly suppress the formation of larger $n$ value phases, but it also enhances the formation of lower $n$ value phases. We found that MAPbl$/ 3 / \mathrm{BA}_{2} \mathrm{Pbl}_{4}$ ratio of $0.6(n=2)$ and $1.3(n=$ 3) can make best compromise for this dilemma and leads to improved phase purity."

"However, it's worth to note that the indicated $\mathrm{MAPb}_{3} / \mathrm{BA}_{2} \mathrm{Pbl}_{4}$ ratio $(0.6$ for $n=$ 2 and 1.3 for $n=3$ ) cannot guarantee the successful preparation of thin films with qualified phase purity. In fact, even under the optimal MAPbl $3 / \mathrm{BA}_{2} \mathrm{Pbl}_{4}$ ratio, only $10-15 \%$ prepared quasi-2D thin films exhibit qualified phase purity. The phase purity of thin films was characterized from parallel samples by using XRD and room-temperature PL measurements. The selected thin films with qualified phase purity were further investigated by the temperature-dependent PL spectroscopy for analyzing exciton-phonon coupling."

2) The SEM morphologies of $n=1$ and $n=3$ looks similar while $n=2$ looks different from the others. Please compare them on the same scale and describe the reasons for the difference in the morphologies of these LD samples.

We thank the reviewer very much for this comment. After rechecking the original files from the SEM station, we found that several graphics (including the $n=2$ graphic we used in Supporting Information of initially submitted version) were mixed up due to the similar naming in identical file folder. We have replaced the misused graphic (Figure S2) with correct one in Supporting Information (Section S2, page 5). All SEM graphics illustrated in Supporting Information have been cross-checked and are obtained under similar condition and on same scale. We sincerely apologize for this careless mistake. 
3) The authors considered the LE emitting states as STE or DE in this study based on some earlier reports. These low energy emitting states have also been proposed as phonon replicas or bi-excitons and triplet excitons in some other studies. The authors should describe clearly on what basis they have ruled out the assignments to bi-excitons (Opt. Express 2010, 18, 59125919, Phys. Rev. B. Condens. Matter Mater. Phys. 200470 205330) or triplet excitons (JPCL 2021, 12, 8, 2133-2141) in this study.

We thank the reviewer very much for this comment. In order to rule out the possibility of biexciton for assignment of LE1 and LE2 bands, we performed the excitation intensity dependent PL experiments at selected temperatures (150K and 20K) for identify LE1 and LE2, respectively. Please note that our excitation source is incoherent Xe lamp (with a monochromator) instead of laser, the excitation beam illuminates samples with unfocused beam profile, we are not capable to measure and tune the absolute value of excitation density. Thus, we performed these experiment in a relative way, i.e. reducing the excitation intensity with neutral density filters of different $O D$ values $(0,0.3,0.4,0.5,0.6$, $0.8,1.0,1.5,2.0$ and 3.0 ), which correspond the relative excitation intensity of $1,0.501,0.398,0.316,0.251,0.158,0.1,0.0316,0.01$ and 0.001 , respectively.
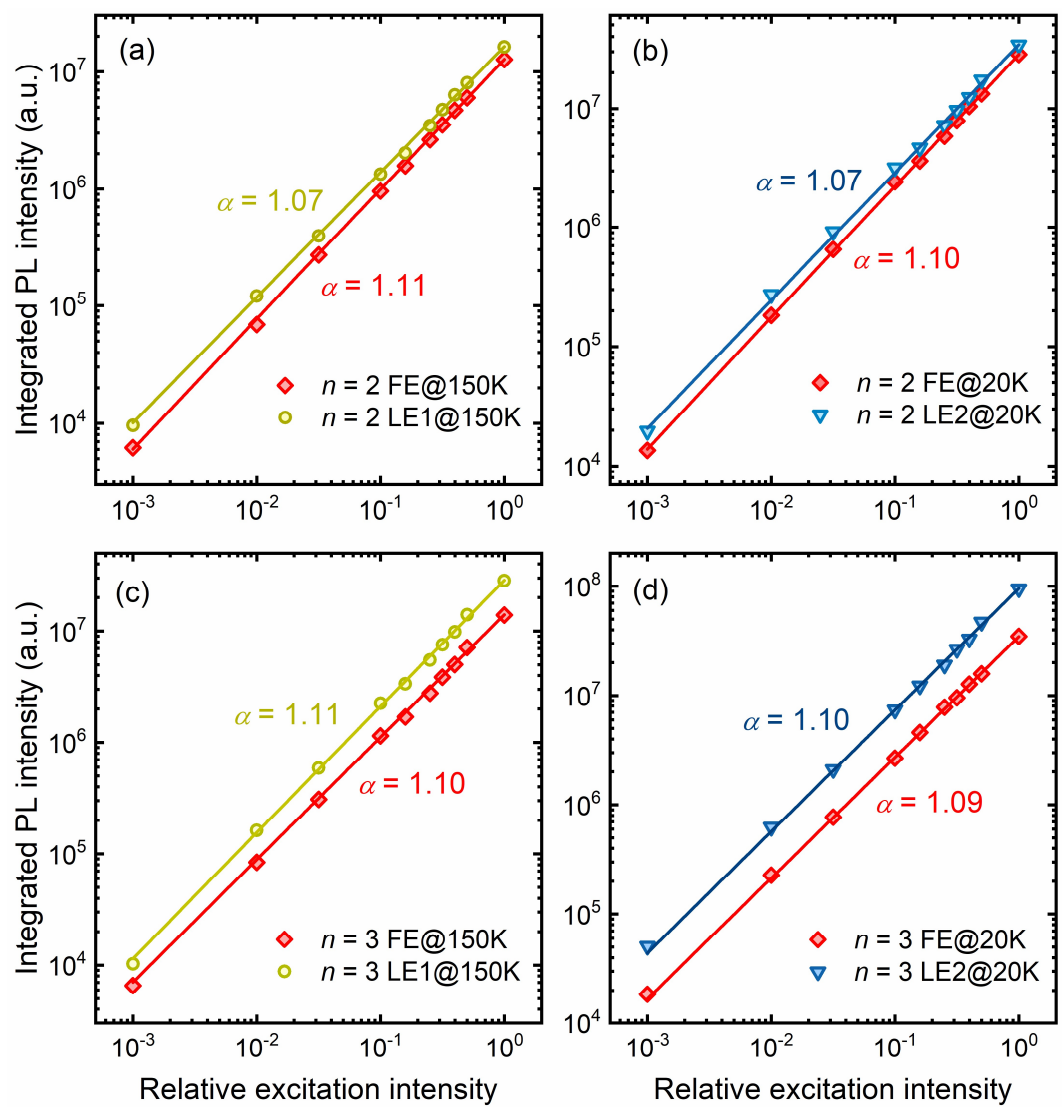

Figure R4. The excitation intensity dependence of emission intensity (integrated spectra) for $n$ $=2$ (upper panel) and $n=3$ (bottom panel) at 150K (left column) and 20K (right column). The power law $\left(I \propto \mathrm{P}^{\alpha}\right)$ fitting are displayed together with the fitted slope $(\alpha)$ values. 
The resulted excitation intensity dependence of integrated emission intensity is displayed in Figure R4. We fitted the integrated PL spectra to a power law $(I \propto$ $\mathrm{P}^{\alpha}$ ) while the resulted $\alpha$ values are summaried in Table R1. As can be seen, the intensity of both FE, LE1 and LE2 increases linearly with the excitation power $(\alpha \approx 1)$, which provides solid evidence to rule out the possibility of biexciton for LE bands. The slight higher $\alpha$ values for both FE, LE1 and LE2 bands may originate from the systematic error of OD values for the ND filters.

Table R1. Fitted slope values $(\alpha)$ for excitation intensity dependence of integrated PL spectra.

\begin{tabular}{|l|l|l|l|l|}
\hline & & FE & LE1 & LE2 \\
\hline \multirow{2}{*}{$n=2$} & $150 \mathrm{~K}$ & 1.07 & 1.11 & \\
\cline { 2 - 5 } & $20 \mathrm{~K}$ & 1.07 & & 1.10 \\
\hline \multirow{2}{*}{$n=3$} & $150 \mathrm{~K}$ & 1.11 & 1.10 & \\
\cline { 2 - 5 } & $20 \mathrm{~K}$ & 1.10 & & 1.09 \\
\hline
\end{tabular}

On the other hand, the most direct way to exclude the existence of triplet excitons is PL lifetime measurement at specific temperature. Unfortunately, the corresponding instrument (TCSPC or steak camera) has not been available yet in our laboratory. The reported triplet emission spectra show comparable linewidth with singlet excitons (i.e. free exciton described in manuscript). Meanwhile, triplet emission shows evident spectral narrowing with temperature decreasing due to the suppression of phonon coupling. However, as illustrated in Figure R5, no characteristic spectral narrowing (like singlet FE) can be observed for LE1 and LE2 bands of LD thin films ( $n=2$ and 3). Therefore, we think the LE bands we observed are unlikely to be the triplet emission.
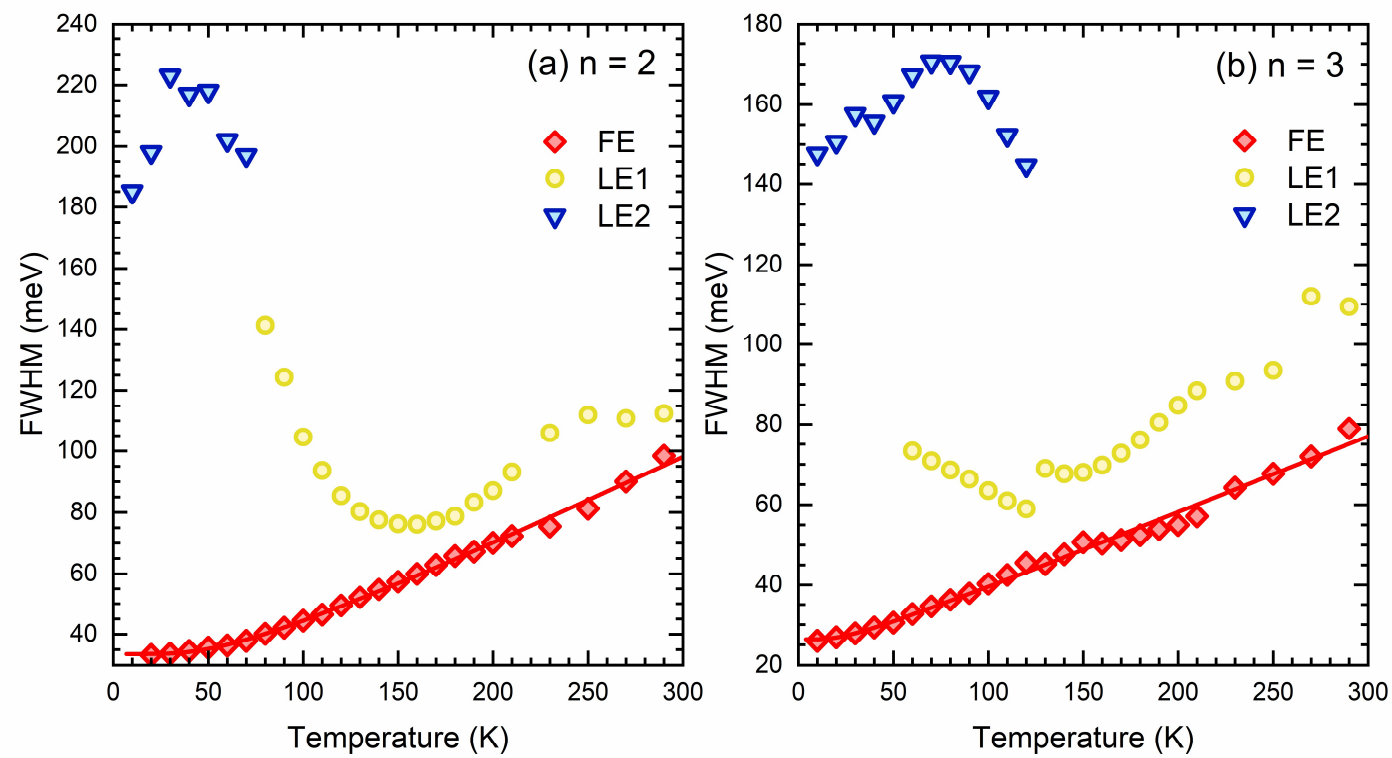

Figure R5. The multi-peak (Gaussian) fitting estimated emission spectra linewidth of FE, LE1, and LE2 bands for LD thin films $n=2$ (a) and $n=3$ (b). 
We implemented the additional information on Page 16: "Last but not least, the low energy PL bands of low-dimensional perovskite have been reported as emission of biexcitons ${ }^{66,67}$ or triplet free excitons. ${ }^{68,69}$ For excluding the possibility of biexcitons, excitation intensity dependent PL measurements were performed on quasi-2D thin films ( $n=2$ and 3 ) at $150 \mathrm{~K}$ and $20 \mathrm{~K}$ for LE1 and LE2 band, respectively. As illustrated in Figure S20 and Table S4, the intensity of LE bands increases almost linearly with excitation intensity $(\alpha \approx 1$ for IPL $\propto$ $\left.P_{E X}{ }^{\alpha}\right)$, which rules out the assignment of biexcitons for observed LE bands. Moreover, the reported emission band of triplet FE in low-dimensional perovskite shows evident spectral narrowing with temperature decreasing, ${ }^{68,69}$ which is obviously rather different from what we observed (see Figure S21) for LE bands of quasi-2D thin films." The corresponding data are illustrated in Supporting Information (Section S12 and S13).

4) The produced phase pure thin films show existence of free excitons, localized excitons and defects. What is the effect of defect concentration on the estimated LO and AO phonon coupling parameters?

We thank reviewer for this important and forward-looking question. It's wellknown that density of structural defects greatly limits performance of semiconductor optoelectronic devices. In particular, the carrier recombination and charge transport is substantially affected by defects in perovskite thin film. However, to the best of our knowledge, the effect of defect concentration on exciton/electron - phonon coupling has not been described anywhere yet. Actually, this is also the important topic in our group, we are currently trying to control the defect density in LD perovskite thin films by changing grain size or performing proper post-treatment, and will further analyze the exciton/electron - phonon coupling in the next step as we described in the present work. We will report the new knowledge once we obtain the reliable results.

Did the authors perform these experiments at slightly different precursor ratios without losing the required phase purity? The authors should provide error bars for all the quantitative parameters by performing experiments at slightly different precursor ratios.

We thank reviewer for the suggestion. As we mentioned above, all thin film samples with qualified phase purity were selected among parallel prepared samples (with the optimal MA/BA ratios) by using XRD and room-temperature PL measurements. As reviewer suggested, we performed implemented experiments and corresponding characterization, which slightly changed the MA/BA ratios ( 0.55 and 0.65 for $n=2,1.25$ and 1.35 for $n=3$ ) and prepared 20 parallel samples for each MA/BA ratios to select additional samples with qualified phase purity. It is found that slight drifting from the optimal MA/BA ratios ( 0.6 for $n=2$ and 1.3 for $n=3$ ) leads to less proportion of phase purity 
qualified LD thin films among all newly prepared samples. For positive deviating from the optimal MA/BA ratios ( 0.65 for $n=2$ and 1.35 for $n=3$ ), the emission of larger $n$ value ( $n=3$ and $n=4$, respectively) appears as shoulder in roomtemperature PL spectra. Meanwhile, the lower MA/BA ratios ( 0.55 for $n=2$ and 1.25 for $n=3)$ lead to significant enhancing of phases with lower $n$ value $(n=1$ and $n=2$, respectively).

Nevertheless, we can still select at least one phase purity qualified thin film by using XRD and room-temperature PL measurements from 20 newly prepared parallel samples for each MA/BA ratios. The selected additional samples have been further investigated by temperature-dependent PL spectroscopy as described in manuscript (Figure R6). The fitted quantitative parameters (fitting of FE linewidth, FE peak wavelength, integrated intensity of FE and LE bands) of additional samples and our initially examined samples lead to average value and standard deviation for each parameters as listed in corresponding tables of manuscript (Table $1-3$ ). It can be seen that majority of quantitative fitting parameters show $10-15 \%$ standard deviation, which indicates the experimental repeatability and reliability for our data.
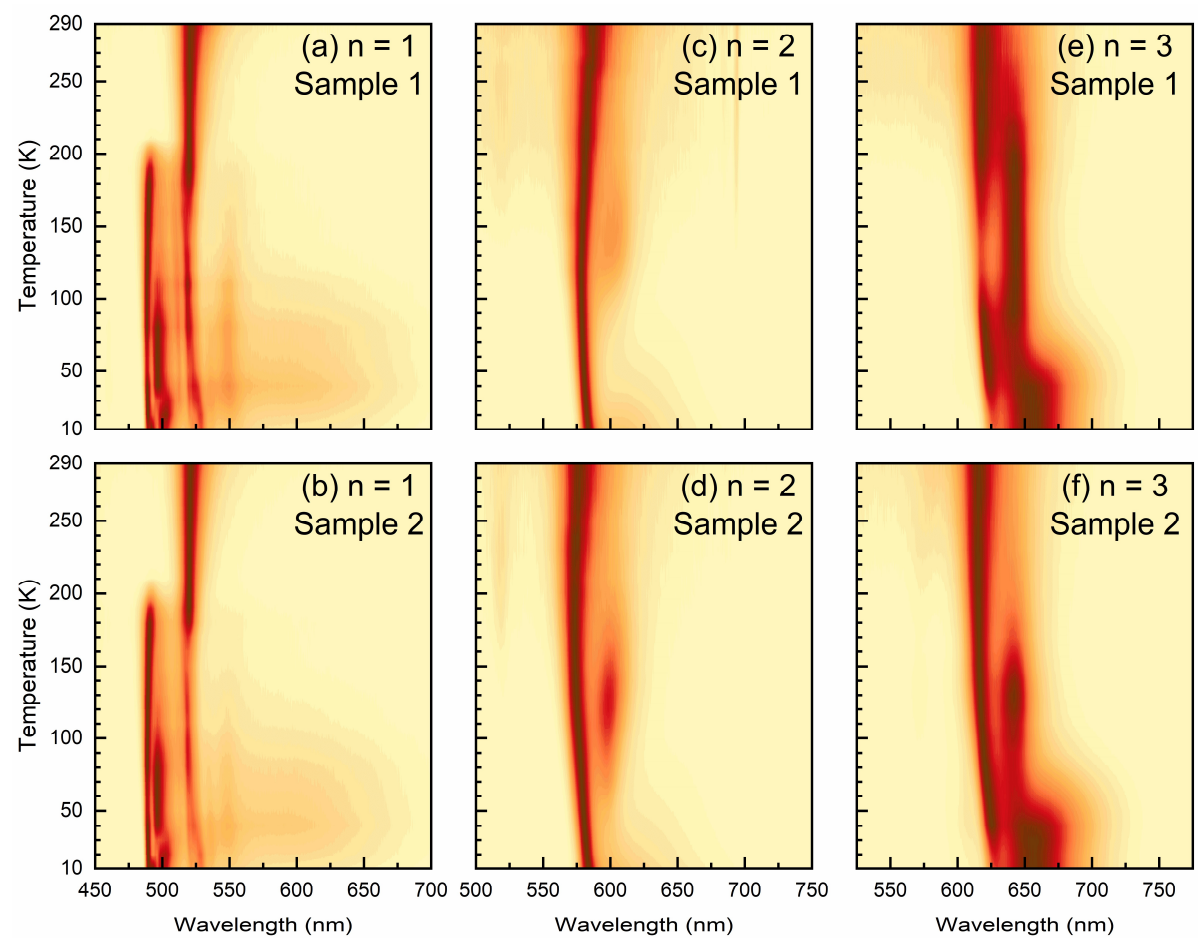

Figure R6. Pseudocolor plots of temperature-dependent photoluminescence of additional phase purity improved $2 \mathrm{D}$ and quasi-2D BA $2 \mathrm{MA}_{n-1} \mathrm{~Pb}_{n} \mid 3_{n+1}(n=1-3)$ thin films.

The related details have been implemented on manuscript page 6: "Beside thin films described here, two additional thin films with qualified phase purity were further prepared for each $n$ values. For quasi-2D ( $n=2$ and 3$)$ thin films, the 
additional thin films were prepared by slightly change the precursor ratios without losing the required phase purity. Temperature-dependent PL spectra of additional thin films are displayed in Figure S8, further quantitative analysis was conducted for all thin films to provide average value and standard deviation of all quantitative fitting parameters described in following sections (original fitting parameters see Table S1 - S3)." The fitted quantitative parameters for all parallel samples are provided in Supporting Information (Section S5).

We also added details in caption of Table $1-3$ as "The average values and standard deviation of each parameter are estimated by analyzing temperaturedependent PL spectra of 3 parallel thin films for each $n$ values."

5) In Figure 4, potential curves are shown in the insets. However, considering these LD perovskites are semiconductors in nature, the way of presenting the relaxation processes should be changed to a semiconducting style rather than a molecular style.

We thank the reviewer for this comment. We've revised the energy level diagram (see Figure 4) to illustrate the relaxation paths of LD perovskite in semiconductor style, which is referred to the published cases such as Acc. Chem. Res., 2019, 52, 3188-3198 and Nat. Mater., 2017, 16, 115-120.

\section{Reviewer 2}

The results reported in this manuscript are interesting. The manuscript is recommended to be published. The question is: how light intensity affects the bandgap?

We thank reviewer for the positive evaluation. Regarding the question, our response is as followed. The blue-shifting of PL spectra (i.e. increasing bandgap) with increasing excitation intensity has been intensively reported for perovskite thin films, which could be attributed to the band filling effect of carriers (Nat. Photonics, 2014, 8, 737-743). However, such bandgap increasing due to band filling effect can only be observed under strong excitation density. As we described in Supporting Information (Section S1), all PL measurements described in this work were performed with incoherent light source with unfocused beam profile, i.e. Xe lamp with monochromator, which leads to low excitation density on samples. The band filling effect is not likely to be observed under such excitation conditions.

In order to exclude the band filling effect experimentally, we performed excitation intensity dependent PL measurements on prepared $2 \mathrm{D}$ and quasi-2D 
thin films. As can be seen in Figure R7, for all measured thin films, the center wavelength of free exciton (i.e. bandgap) keeps constant in whole excitation intensity range, which excludes the existence of band filling effect under our employed experimental conditions.
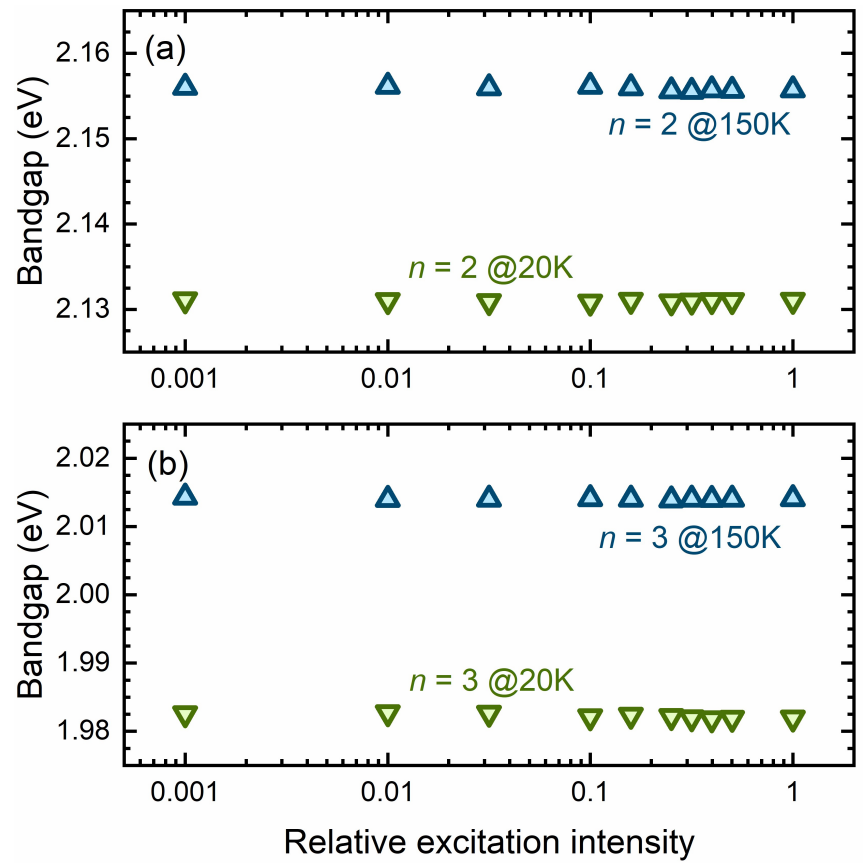

Figure R7. The excitation intensity dependent bandgap (i.e. center wavelength of free exciton $\mathrm{PL}$ peak) of quasi-2D thin films at $150 \mathrm{~K}$ and $20 \mathrm{~K}$.

We implemented description on Page 13: "It is worth to note that the band filling effect has been intensively reported, which increases the band gap of perovskite thin films with increasing excitation intensity. ${ }^{59-61}$ Excitation intensity dependent PL measurements were performed on prepared 2D and quasi-2D thin films at $150 \mathrm{~K}$ and $20 \mathrm{~K}$. As illustrated in Figure S19, no drifting of bandgap can be observed, which excludes the existence of band filling effect and certifies the reliability of the analysis described in this section." The corresponding data are displayed in Supporting Information (Section S11). 


\section{List of Major Changes (see also marked-up version of manuscript)}

1) As editor suggested, we reformatted the title in both the main manuscript and supporting information, i.e. with the first letter of each principal word capitalized.

2) We modified and resized the TOC graphic to $2 \times 2$ inch.

3) We checked all cited references and fixed their style to use JPCL formatting.

4) We reformatted the whole manuscript to fulfill the requirement of JPCL.

5) The details for preparing the quasi-2D thin films with improved phase purity was implemented in Supporting Information (Section S1).

6) We replaced the misused SEM graphic in Supporting Information (Section S2).

7) We excluded the involving of biexciton and triplet free exciton by performing additional experiment and analysis.

8) We repeated temperature-dependent PL measurements and data analysis on newly prepared additional samples, which provides standard deviation for all quantitative fitting parameters.

9) The energy diagram in Figure 4 has been revised to describe the relaxation paths of LD perovskite in a semiconductor style.

10) The bandgap changing due to the band filling effect was excluded, corresponding information is implemented in manuscript.

11) We revised several minor issues regarding the language, which have been marked in manuscript as red color. 